\title{
O USO DO COMPUTADOR EM PROGRAMAS DE REABILITAÇÃO NEUROPSICOLÓGICA
}

\section{The Use of the Computer in Neuropsychological Reabilitation Programs}

\author{
Erika Crisóstomo Albuquerque \\ EdsonEmílio Scalabrin ${ }^{2}$
}

\section{Resumo}

Este trabalho se constitui em um artigo de revisão no qual são descritas as práticas de desenvolvimento de reabilitação cognitiva que utilizam metodologias computacionais em pessoas com desordem cognitiva ou dano cerebral, especialmente aquelas destinadas a portadores da Demência de Tipo Alzheimer. Os resultados revelam que a incipiência de projetos baseados em recursos computacionais em torno dessa patologia talvez se deva ao fato de que, ainda hoje, pouco se conhece sobre as causas da doença e de como se realiza o processamento mental de informações nesses pacientes. Isso indica um campo de estudo em plena expansão que irá requerer a interdisciplinaridade das ciências que desejam contribuir com a construção de novos instrumentos de pesquisa e reabilitação cognitiva do Alzheimer.

Palavras-chave: Reabilitação cognitiva; Inteligência artificial; Alzheimer.

\section{Abstract}

This work constitute an revision paper in which are describes the development of cognitive rehabilitation practices that uses computes methodologies in people with cognitive disorder or a cerebral damage, especially, those fated with bearers of the insanity of the type Alzheimer. The results reveal that the beginning for the accomplishment of computes projects around that pathology, maybe be due to the fact that, even today, little it is known about cause of the disease and as it takes place the information mental process in these patient. That indicates a study field in the middle of expansion that will go apply science's interdisciplines that they want to contribute with the construction of new research instruments and Alzheimer's cognitive rehabilitation.

Keywords: Cognitive rehabilitation; Artificial intelligence; Alzheimer.

Psicóloga. Mestranda em Tecnologia em Saúde pela Pontifícia Universidade Católica do Paraná. Curitiba, PR. e-mail: erikaleme@gmail.com

Professor-Doutor dos cursos de Mestrado e Doutorado da Pontifícia Universidade Católica do Paraná. Cientista da Computação. Endereço para correspondência: Pontifícia Universidade Católica do Paraná - PUCPR, Centro de Ciências Exatas e de Tecnologia, Departamento de Informática. Curitiba, PR. e-mail: scalabrin@ppgia.pucpr.br 


\section{Introdução}

A ampliação do uso da tecnologia em diversas áreas do conhecimento vem crescendo nos últimos anos e possibilitando um considerável progresso nas descobertas científicas, particularmente nas relacionadas às ciências da saúde. Dessa maneira, a informática tem permitido a ampliação das possibilidades diagnósticas e terapêuticas, pois, por meio de seus recursos computacionais (equipamentos, dispositivos e programas, entre outros), facilita o acesso à reabilitação de pacientes com diferentes tipos de danos cerebrais sejam eles de ordem cognitiva ou motora.

No Brasil, os trabalhos voltados ao uso de tecnologias computacionais na reabilitação cognitiva ainda é embrionário. Cabe ressaltar que as contribuições reveladas nesse artigo exploraram esse potencial em diferentes tipos de desordens cognitivas, oferecendo significativas oportunidades aos pacientes perante situações do dia-a-dia. Contudo, a versatilidade desses estudos parece não se aplicar às particularidades do comprometimento cognitivo que afeta o idoso especialmente quando se trata de quadros degenerativos, como é o caso da doença de Alzheimer.

É dentro dessa perspectiva que o presente artigo se propõe a avaliar o desenvolvimento das pesquisas sobre o uso de tecnologias na reabilitação de indivíduos com déficits cognitivos, especialmente aqueles acometidos pelo Alzheimer, bem como as ferramentas computacionais disponíveis para esse fim.

Para tanto, o método de estudo compreendeu, além do trabalho de Costa (2000) como principal referência, pesquisas na biblioteca BIREME, nas bases de dados LILACS, MEDLINE (1996 a 2006) e SCIELO. As palavras-chave incluíram sempre os termos reabilitação cognitiva ou reabilitação neuropsicológica e computador. O uso dos descritores "terapia cognitiva", "terapia assistida por computador" e "doença de Alzheimer", em português e em inglês, associadas ou não quando necessário, complementaram as buscas que indicaram ao todo 31 artigos relacionados ao título. Os trabalhos que envolviam diretamente o uso do computador em atividades de reabilitação neuropsicológica, sobretudo àquelas que envolvam pacientes com Alzheimer, são mencionados no decorrer desse artigo.

A estrutura do presente trabalho está organizada em três momentos: um primeiro, onde são descritos brevemente alguns dados obtidos sobre as características, evolução e epidemiologia do Alzheimer. Em seguida, o texto procura esclarecer quais as principais abordagens oferecidas pela psicologia para o entendimento da cognição humana, o que vem a ser um processo de reabilitação cognitiva e quais os procedimentos normalmente aceitos pela comunidade da saúde para sua realização. Finalmente, o artigo aborda o modo como a reabilitação cognitiva vem sendo alvo do interesse de pesquisadores de diferentes áreas, sobretudo da ciência da computação e o quanto as possibilidades oferecidas pela Inteligência Artificial se mostram adequadas para esse fim.

\section{Alzbeimer}

Embora diferentes pesquisas estejam sendo desenvolvidas no mundo inteiro, a doença de Alzheimer continua sendo um grande mistério para a ciência. Sua causa é ainda desconhecida e o diagnóstico, mesmo adotando critérios padronizados internacionalmente, não dispõe de exames que a comprovem com precisão, sendo definitivo apenas mediante a análise histopatológica do tecido cerebral post-mortem (Gallucci; Tamelini e Forlenza, 2005).

O termo Demência de Tipo Alzheimer (DTA), como é adotado no DSM-IV (1994) $4^{\text {a }}$ edição do manual diagnóstico e estatístico dos transtornos mentais, a um distúrbio cerebral degenerativo progressivo, caracterizado por deficiência da memória e de outras funções cognitivas que resultam em alterações comportamentais que interferem na vida social do individuo, levando-o em último estágio à incapacidade irreversível de ação.

O curso clínico da DTA é caracterizado pelo enfraquecimento gradual da memória, da atividade intelectual e da capacidade de executar as atividades da vida diária. Tipicamente, a memória recente (a que nos fornece informações sobre fatos ocorridos não mais que nas últimas seis horas) parece mais deficiente do que a memória remota (lembranças de dias e anos anteriores).

Embora no primeiro estágio da doença as mudanças possam não ser reconhecidas pela maioria das pessoas, na medida em que o distúrbio começa a progredir, fatores cognitivos, comportamentais e psicológicos são responsáveis pelo grau da doença e da capacidade de o indivíduo interagir socialmente. Em seu estágio final, as capacidades para raciocinar e se orientar no mundo ficam drasticamente deterioradas (Magnié e Thomas, 1998). 
Levando em conta a relação diretamente proporcional que a doença mantém com o aumento da idade, bem como a perspectiva do crescente aumento populacional de idosos nas próximas décadas, isso nos revela que a DTA pode ser encarada como sério problema de saúde pública, além de um importante modelo para os estudos neurocognitivos sobre o funcionamento do cérebro.

\section{Reabilitação cognitiva}

Existem diversas abordagens científicas que nos proporcionam referenciais úteis para pensarmos sobre a natureza e o desenvolvimento da cognição. Entretanto, é a teoria do processamento da informação que, na visão de Flavell, Miller \& Miller (1999), encontramos uma das principais estratégias da psicologia para o estudo dos processos cognitivos, particularmente aqueles ligados à memória. Ela concebe a mente humana, análoga a um computador digital, como um sistema cognitivo complexo, que manipula ou processa as informações que são oriundas do ambiente ou já estão armazenadas dentro do sistema. Desse modo, o processamento ocorre à medida que: a informação é capturada, transformada em uma representação mental, comparada com informações já processadas, recebe um significado e é posteriormente armazenada. "Uma seqüência de armazenamento e recuperação que, à medida que é praticada e executada com mais eficiência, se torna automatizada." (Flavell, Miller \& Miller, 1999, p. 191).

Outra visão sobre a cognição nos é fornecida por Gazzaniga e Heatherton (2005), que a definem como uma complexa coleção de habilidades mentais que permitem que o homem compreenda e relacione-se com o mundo durante o seu curso de vida, por meio de funções que incluem a percepção, atenção, compreensão, aprendizagem, memória e raciocínio, entre outras.

No dano cerebral, uma ou várias destas atividades podem se tornar deficientes e sua reabilitação deve compreender estratégias terapêuticas específicas para cada tipo de deficiência detectada. Nesse contexto, a reabilitação cognitiva se define como a "aplicação de procedimentos e utilização de técnicas de apoio para que a pessoa com déficits cognitivos possa retornar de maneira segura, produtiva e independente a suas atividades cotidianas" (Matter, 2003, p. 12). Para tanto, alguns elementos são importantes na hora de planejar, desenvolver e avaliar um programa de reabilitação: compreender todos os processos que envolvem o dano cerebral e sua evolução, identificar o estilo de vida anterior e realizar uma avaliação completa das capacidades cognitivas preservadas e alteradas, bem como dos recursos humanos, materiais, ambientais e tecnológicos disponíveis para uma estratégia terapêutica (Matter, 2003).

Comumente, a reabilitação cognitiva ocorre de modo multidisciplinar. Inicia-se após análise médica do neurologista que identifica o dano neuronal em termos anatômicos e fisiológicos. Posteriormente, o neuropsicólogo concentra sua atenção na avaliação das deficiências cognitivas e afetivas efetivamente provocadas pelo dano. Finalmente, traçada a estratégia de reabilitação cognitiva, é necessário acionar um conjunto de especialistas em geriatria e gerontologia, que envolverá profissionais da enfermagem, psicologia, assistência social, fisioterapia, nutrição e até mesmo a advocacia para contribuir com a manutenção da homeostase física, mental e social do paciente (Costa, 2000).

Ávila (2003) nos informa ainda que, especialmente na DTA, aliada ao tratamento farmacológico, a metodologia de reabilitação cognitiva utilizada normalmente engloba medidas de adaptação e organização do ambiente a fim de facilitar as atividades da vida diária, modificação das interações sociais do doente em especial com seus familiares, exercícios de linguagem e tratamento psicoterapêutico, o que forma um composto de práticas particularmente chamado por especialistas de reabilitação neuropsicológica.

\section{Tecnologias computacionais e reabilitação cognitiva}

É notório, nas últimas cinco décadas, a forma heterogênea como diversas ciências vêm buscando compreender os mistérios da mente humana. Essa interdisciplinaridade de áreas na evolução das pesquisas sobre o processamento da mente culminou no surgimento de uma nova disciplina de estudos: a ciência cognitiva. De um modo geral, a ciência cognitiva reúne modelos e técnicas experimentais de áreas como a psicologia, engenharia, neurociência, lingüística e a computação, entre outras, para tentar construir teorias verificáveis a respeito dos processos mentais. 
Quando falamos de tecnologias computacionais voltadas para a reabilitação cognitiva, é inevitável mencionar os modelos computacionais desenvolvidos pela Inteligência Artificial. A Inteligência Artificial, orientada pelos pressupostos da ciência cognitiva, caracteriza-se basicamente pelo objetivo de aplicar tal conhecimento na construção de máquinas capazes de interagir com o meio mediante uma mente inteligente. Suas aplicações podem ser vistas de duas maneiras: em abordagens que utilizam aspectos psicológicos e processos algorítmicos para modelar o comportamento inteligente em agentes ${ }^{3}$ computacionais, ou em sistemas inteligentes baseados em modelos de funcionamento do cérebro, dos neurônios e das conexões neurais (Russel, 2004).

A utilização da Inteligência Artificial na produção de artefatos tecnológicos para a área de saúde teve grande expansão na década de 80, especialmente com o surgimento dos sistemas especialistas baseados em recursos computacionais com ampla capacidade de reunir e analisar grandes volumes de dados médicos que estariam envolvidos na tomada de decisões diagnósticas. Atualmente, é na Inteligência Artificial que encontramos a crescente preocupação da informática na elaboração de recursos computacionais que colaborem com programas de reabilitação na área da saúde.

Especialmente quando falamos em reabilitação cognitiva, as tecnologias se expandem na direção da criação de agentes computacionais que possam interagir com os pacientes de modo a tornálos mais motivados e comprometidos com o tratamento. Nesse sentido, Costa (2000) esclarece que programas de reabilitação com abordagem tecnológica devem desenvolver as potencialidades e diminuir as limitações das pessoas, buscando estimular suas potencialidades físicas, mentais e sensoriais. Para tanto, os softwares e os dispositivos periféricos utilizados devem ser ajustados e adaptados para satisfazer amplamente as necessidades físicas, cognitivas e psicológicas do paciente. Isso requer uma preocupação cuidadosa na elaboração das interfaces a serem utilizadas, "considerando a acessibilidade ao equipamento, os aspectos posturais, o quadro clínico que o paciente apresenta e suas implicações, as adaptações necessárias e o interesse e as demandas do paciente" (Watanabe, Tsukimoto, D. R., \& Tsukimoto, G. R. 2003, p. 19).
Embora a exploração do computador na Reabilitação cognitiva estar em seus estágios iniciais de pesquisa, principalmente devido aos altos custos e à complexidade dos mecanismos e equipamentos envolvidos, os trabalhos de Costa $(2000,2004)$, pioneiros no Brasil nesse campo de estudo, indicanos o uso de tecnologias inteligentes como um campo promissor em plena expansão, especialmente no que se refere ao uso dos recursos oferecidos pela Realidade Virtual, um tipo particular de tecnologia que "envolve equipamentos especiais e apóia o desenvolvimento e a exploração de aplicações compostas por cenas e situações simuladas em computadores" (Costa, 2000, p. 52).

Para a tecnologia de Reabilitação cognitiva, os resultados desta revisão revelam que os estudos feitos até o momento apontam para duas vertentes da tecnologia cognitiva: uma que se preocupa em desenvolver recursos computacionais para as deficiências cognitivas causadas por dano cerebral traumático, e outra envolvida na elaboração de sistemas voltados às pessoas com desordens psiquiátricas. Na primeira linha de pesquisa, encontramos os trabalhos de Santarosa, Moori \& Franco, (1996); Bolognani et al. (2000); Drummond, Costa \& Carvalho (2002); Wauke, Carvalho \& Costa (2005); Santana, Ramirez \& Ostrosky-Sólis (2004).

No segundo grupo, bem mais expressivo em número de publicações, citamos os trabalhos de Costa (2000); Costa, \& Carvalho (2004); Wright, J. H. et al. (2005); Hofmann, M. et al. (2003); Perpiñá, Baños, Botella \& Marco (2001); Grohman, Fals-Stewart \& Donnelly (2006); Van den Berg S., Shapiro, Bickerstaffe \& Cavanagh (2004); Proudfoot et al. (2004); Grime (2004); Cavanagh \& Shapiro (2004); Gega, Marks \& Mataix-Cols (2004); Wiederhold et al. (2002); Riva, Bacchetta, Cesa, Conti, \& Molinari (2002); Kaltenthaler et al. (2002); Jang et al. (2002); Carlbring, Ekselius, \& Andersson (2003); Baños et al. (2002); Célio et al. (2000).

No primeiro grupo, de um modo geral, as práticas terapêuticas desenvolvidas por meio do computador revelam que houve melhora nos pacientes das habilidades motoras e cognitivas, aumento da motivação e comprometimento com o tratamento, o que repercutiu de forma positiva no processo de reabilitação como um todo. Entretanto, embora as vantagens que um ambiente computacional oferece sejam percebidas na maioria das pesquisas, em alguns

O termo agente na computação é utilizado para descrever amplamente processos de hardware ou software. 
casos, o computador ainda é descrito apenas como uma ferramenta coadjuvante no processo, não sendo atribuído a ele, ou a suas características, nenhum progresso do tratamento. Essa postura, aliada às dificuldades para adaptação de interfaces adequadas ao grau de comprometimento físico e cognitivo que acometem pacientes com deficiências cognitivas causadas por dano cerebral traumático, talvez explique o reduzido número de trabalhos que avaliem o uso do computador como recurso de reabilitação cognitiva nessa modalidade.

$\mathrm{Na}$ segunda vertente de estudos, os resultados demonstram que as terapias cognitivas desenvolvidas por intermédio do computador se mostram tão ou mais eficazes quanto os modelos de reabilitação tradicionais e ainda podem apresentar a vantagem de assegurar privacidade $\mathrm{e}$ confidencialidade, diminuir custos e conseqüentemente melhorar o acesso à terapia cognitiva, em especial nas patologias associadas a quadros de fobia, ansiedade, síndromes do pânico, transtornos obsessivos compulsivos, distorções alimentares, dependência química e depressão.

Nesses casos, o uso da Realidade Virtual, como propõe Costa (2000), mostrou-se uma tecnologia realmente promissora e poderosa, deixando de ser apenas alvo de interesse de pesquisadores acadêmicos e vislumbrando o interesse comercial de empresas produtoras de tecnologia médica, pois possibilita uma sofisticada interação entre o paciente e o computador ao explorar situações similares às experimentadas no mundo real, fornecendo amplas aplicações na clínica psicológica desenvolvida por intermédio de técnicas comportamentais (Riva et al., 2002).

Em relação ao foco principal deste estudo, os trabalhos voltados à reabilitação da doença de Alzheimer, apenas uma referência foi encontrada, em Hofmann et al. (2003). Trata-se de uma experiência com quatro pacientes diagnosticados como prováveis portadores da doença de Alzheimer, submetidos a um treinamento das habilidades para executar as atividades da vida diária com um programa de computador. Foram percebidos no estudo, além dos resultados neurológicos da ativação emocional, níveis altos de motivação e aceitação positiva do treinamento, o que contribuiu de maneira favorável ao tratamento.

Assim, os trabalhos citados revelaram que a utilização do computador como recurso para as práticas de reabilitação cognitiva está aos poucos sendo incorporado à área de saúde, à medida que os resultados demonstram significativas melhoras nos aspectos cognitivos, motores, de comunicação e de acesso a informações, possibilitando maior autonomia e interesse dos pacientes, e até mesmo permitindo que o indivíduo retome seu lugar na família e na sociedade.

\section{Conclusões}

As descobertas científicas produzidas no âmbito da ciência da computação ainda são incipientes no que tange à reabilitação cognitiva de portadores de DTA. Isso se deve, em parte, ao fato de que o processamento mental, bem como as causas que ocasionam os déficits cognitivos em idosos demenciados, não está ainda totalmente esclarecido para a ciência.

Entretanto, o surgimento de novos campos de pesquisa a cada ano confirma a progressiva tendência tecnológica em concentrar esforços para apoiar práticas em saúde, sobretudo àquelas que envolvam atividades de reabilitação cognitiva por meio do computador, sejam elas voltadas às deficiências cognitivas causadas por dano cerebral traumático ou desordens psiquiátricas.

Está claro que um componente importante na implementação de estratégias de reabilitação cognitiva baseada no computador deve levar em conta os padrões ergonômicos do projeto da interface, i.é., deve primar por tecnologias moldadas de acordo com as habilidades e necessidades específicas do paciente. Nesse sentido, as contribuições trazidas pelos sistemas baseados em Inteligência Artificial demonstram sua importância não só como parte do processo de reabilitação, mas também permitindo a potencialização de seus resultados.

É possível que as tecnologias baseadas em recursos computacionais desenvolvidas por meio da Inteligência Artificial devam contribuir cada vez mais de maneira significativamente positiva nas atividades diárias de pessoas com déficits cognitivos. Esperamos que, muito em breve, seus artefatos possam disponibilizar agentes inteligentes idealizados não só para a intervenção no processo de reabilitação cognitiva, mas também para atuarem como assistentes pessoais verdadeiramente úteis para uso em qualquer ambiente, favorecendo a autonomia, a liberdade e até mesmo a reintegração de indivíduos à sociedade. 
Um caminho importante a ser percorrido para esse fim está nas descobertas da neurociência acerca da capacidade plástica do cérebro. Nesse sentido, sabemos que os efeitos da influência ambiental na regeneração sináptica de neurônios danificados em pessoas com déficits cognitivos já haviam sido confirmados em práticas de reabilitação cognitiva tradicional, como salienta Ávila (2003). Entretanto, a comprovação desses efeitos em virtude de programas desenvolvidos por intermédio do computador, como foi constatado nos trabalhos citados anteriormente, reforça a crença de que a integração entre áreas como a neurologia, a psicologia, a informática, a educação e a ciência cognitiva, entre outras, é fundamental para avançarmos nesse campo.

Particularmente no domínio de estratégias de reabilitação em indivíduos portadores de Alzheimer, os resultados até aqui indicam um provável caminho no qual os esforços tecnológicos deverão se concentrar: na produção de tecnologias inteligentes que disponibilizem atividades cognitivas que possam restaurar habilidades danificadas e dar novas possibilidades de independência, desenvolvimento e qualidade de vida ao idoso.

Assim, as possibilidades que a ciência da computação oferece rumo à reabilitação cognitiva dos portadores de Alzheimer se revelaram um campo de investigação pouco explorado e consideravelmente relevante e oportuno para o momento, indicando um caminho desafiante a ser trilhado por aqueles que consideram o computador um aliado no propósito de capacitar máquinas a pensar e auxiliar o homem em suas dificuldades.

\section{Referências}

Ávila, R. (2003). Resultados da reabilitação neuropsicológica em paciente com doença de Alzheimer leve. Psiquiatria Clínica, 30(4), 139-146.

Baños, R. M, Botella, C., Perpiñá, C., Alcañiz, M., Lozano, JÁ., Osma, J., et al. (2002). Virtual reality treatment of flying phobia. IEEE Trans Inf Technol Biomed, 6(3), 206-212.

Bolognani, S. P. et al. (2000). Memória implícita e sua contribuição à reabilitação de um paciente amnéstico: relato de caso. Arq Neuro-Psiquiatr, 58(3B), 924-930.
Carlbring, P., Ekselius, L., \& Andersson, G. (2003). Treatment of panic disorder via the Internet: a randomized trial of CBT vs. applied relaxation. J Behav Ther Exp Psychiatry, 34(2), 129-140.

Cavanagh, K., \& Shapiro, D. A. (2004). Computer treatment for common mental health problems. J Clin Psychol, 60(3), 239-251.

Celio A. A., Winzelberg, A. J., Wilfley, D. E., Eppstein-Herald, D., Springer, E. A., Dev, P. et al. (2000). Reducing risk factors for eating disorders: comparison of an Internet and a classroom-delivered psychoeducational program. J Consult Clin Psychol, 68(4), 650-657.

Costa, R. M. E.(2000). Ambientes virtuais na reabilitação cognitiva de pacientes neurológicos e psiquiátricos. Tese de Doutorado em Ciência da Computação, Universidade Federal do Rio de Janeiro, Rio de Janeiro.

Costa, R. M. E. M., \& Carvalho, L. A. V. de. (2004). The Acceptance of Virtual Reality Devices for Cognitive Rehabilitation: a report of positive results with schizophrenia. Computer Methods and Programs in Biomedicine, 73(3), 173-182.

Drummond, R., Costa, R. M. E. M., \& Carvalho, L. A. V. de. (2002). Estimulação Cognitiva de Pessoas com Transtorno Autista através de Ambientes Virtuais. Cadernos do Ime Série Informática, 13, 40-50.

DSM-IV. American Psychiatric Association. (1994). Manual de Diagnóstico e Estatístico de Distúrbios Mentais. Porto Alegre: Artes Médicas.

Flavell, J. H., Miller P. H., \& Miller, S. A. (1999). Desenvolvimento cognitivo. Porto Alegre: Artes Médicas.

Gallucci, N. J., Tamelini, M. G., \& Forlenza, O. V. (2005). Diagnóstico diferencial das demências. Psiquiatria Clínica, 32(3), 119-130.

Gazzaniga, M. S., \& Heatherton, T. F. (2005). Ciência psicológica: Mente, cérebro e comportamento. Porto Alegre: Artmed.

Gega, L, Marks I., \& Mataix-Cols, D. (2004). Computer-aided CBT self-help for anxiety and depressive disorders: experience of a London clinic and future directions. J Clin Psychol, 60(2), 147-157. 
Grime, PR. (2004). Computerized cognitive behavioural therapy at work: a randomized controlled trial in employees with recent stress-related absenteeism. Occup Med (Lond), 54(5), 353-359.

Grohman, K, Fals-Stewart, W. \& Donnelly. K. (2006). Improving treatment response of cognitively impaired veterans with neuropsychological rehabilitation. Brain Cogn, 60(2), 203-204.

Hofmann M., Rösler A., Schwarz W., Müller-Spahn F., Kräuchi K., Hock C. et al. (2003). Interactive computer-training as a therapeutic tool in Alzheimer's disease. Compr Psychiatry, 44(3), 213-219.

Jang, D. P., Ku, J. H., Choi, Y. H., Wiederhold, B. K., Nam, S. W., Kim, I. Y. et al. (2002). The development of virtual reality therapy (VRT) system for the treatment of acrophobia and therapeutic case. IEEE Trans Inf Technol Biomed, 6(3), 213-217.

Kaltenthaler, E., Shackley, P., Stevens, K., Beverley, C., Parry, G., Chilcott, J. (2002). A systematic review and economic evaluation of computerised cognitive behaviour therapy for depression and anxiety. Health Technol Assess, 6(22), 1-89.

Magnié, M. N., \& Thomas, P. (1998). Doença de alzheimer. São Paulo: Andrei.

Matter, C. A. (2003). Introducción a la Rehabilitación cognitive. Avances em Psicologia Clínica Latinoamericana, 21, 11-20.

Perpiñá, C., Baños, R. M., Botella, C., \& Marco, J. H. (2001). La realidad virtual como herramienta terapéutica: Un estudio de caso en las alteraciones de la imagen corporal en los trastornos alimentarios. Rev Argent Clín Psicol, 10(3), 227-241.

Proudfoot, J., Ryden, C., Everitt, B., Shapiro, D.A., Goldberg, D., Mann, A. et al. (2004). Clinical efficacy of computerised cognitive-behavioural therapy for anxiety and depression in primary care: randomised controlled trial. Br J Psychiatry, 185, 46-54.

Riva, G., Bacchetta, M., Cesa, G., Conti, S., \& Molinari, E. (2002). Virtual-reality-based multidimensional therapy for the treatment of body image disturbances in binge eating disorders: a preliminary controlled study. IEEE Trans Inf Technol Biomed, 6(3), 224-234.
Russell, S. J. \& Norvig, P. (2004). Inteligência artificial. Rio de Janeiro: Elsevier.

Santana, D., Ramírez, M., \& Ostrosky-Solís, F. (2004). Novedades en tecnología de rehabilitación: una revisión acerca de la interfaz cerebro-computadora. Revista Neurologia, 39(5), 447-450.

Santarosa, L., Moori, A., \& Franco, B. (1996). Ambientes de Aprendizagem Computacionais para o Desenvolvimento de Jovens Portadores de Paralisia Cerebral. Revista Integração, 7(17), 33-40.

Watanabe, M. K. F., Tsukimoto, D. R., \& Tsukimoto, G. R. (2003). Terapia Ocupacional e o uso do computador como recurso terapêutico. Acta Fisiátrica, 10(1), 17-20.

Wauke, A. P., Carvalho, L. A. V. de, \& Costa, R. M. E. M. (2005). Tratamento de Fobias Urbanas por meio de ambientes virtuais. Arquivos Brasileiros de Psiquiatria, Neurologia e Medicina Legal, 99(4), 5-11.

Wiederhold, B. K., Jang, D. P., Gevirtz, R. G., Kim, S. I., Kim, I. Y., Wiederhold M. D. (2002). The treatment of fear of flying: a controlled study of imaginal and virtual reality graded exposure therapy. IEEE Trans Inf Technol Biomed, 6(3), 218-223.

Wright, J. H., Wright, A. S., Albano, A. M., Basco, M. R., Goldsmith, L. J., Raffield, T. et al. (2005). Computer-assisted cognitive therapy for depression: maintaining efficacy while reducing therapist time. American Journal of Psychiatry, 162(6), 158-164.

Van den Berg, S., Shapiro, D. A., Bickerstaffe, D. \& Cavanagh, K. (2004). Computerized cognitive- behaviour therapy for anxiety and depression: a practical solution to the shortage of trained therapists. J Psychiatr Ment Health Nurs, 11(5), 508-513.

Recebido em: 24/10/2006 Received in: $10 / 24 / 2006$ Aprovado em: 06/12/2006 Approved in: 12/06/2006 